\title{
The podology problems in children and youth in gynecological, pediatric, neurological, and orthopedic perspectives. Diagnosis and therapy of feet deformities. Application of sport in the course of treatment
}

\author{
Tomasz Karski ${ }^{1}$, Jacek Karski ${ }^{2}$, Jan Oleszczuk², Henryka Oleszczuk², Jan Kiljańskii Katarzyna Karska ${ }^{2}$ \\ ${ }^{1}$ Vincent Pol University In Lublin \\ ${ }^{2}$ Medical University in Lublin
}

\begin{abstract}
In last years of the 20th and in beginning of the 21st centuries, we observe the increasing number of children with feet deformities. There are valgus or valgo - planus deformity because of shortening of $\mathrm{m}$. triceps surae and Achilles tendon. The development of these deformities is connected with function - walking, sometimes with incorrect therapy. The primary cause of shortening of flexors of feet and also, of other flexors of other joints, is minimal brain dysfunction (MBD), often in a form of ADHD (attention deficit hyperactivity disorder) - connected with complications during pregnancy and in delivery. The authors described etiopathogenesis of deformities, as well the accompanying deformities in knees and in position of pelvis. In the article there are presented also the methods of physiotherapy.
\end{abstract}

Key words: feet deformities, contractures, minimal brain dysfunctions.

Introduction. Podology in Poland has a long tradition. Prof. Wiktor Dega as well as Prof. Stanisław Piątkowski - the father of Orthopedics in Lublin District (date of grounding of orthopedics in Lublin - 7 7th December 1954) - had been working in the field of podology which resulted in the publication of numerous articles about the subject.

It has been observed that in the 20th and the 21st centuries, the number of children with feet deformities and in need of consultations and treatment has been increasing. In the recent years, 30\% of patients presented in the Lublin Children's Hospital and in Outpatients Clinic admitted for further treatment had problems related to feet.

Flat foot (pes planus) and valgus deformity. Etiopathogenesis. The causes of flat and valgus deformity can typically be subdivided into 3 groups: $1 /$ genetically conditioned (maternal or paternal) which is seldom, 2/ general laxity - which is frequent, 3 / in children with minimal brain dysfunctions (MBD, ADHD) or even in children with minimal or full cerebral palsy connected with spasticity which is frequent (CP). (Fig. 1)

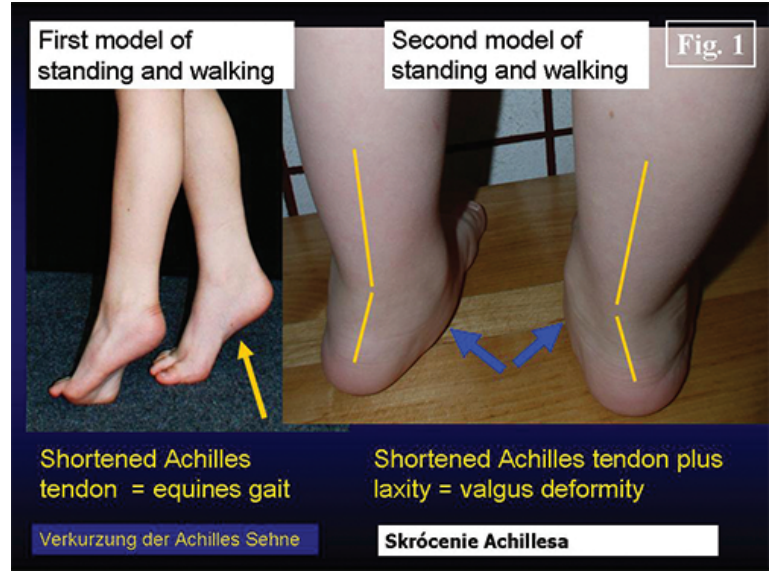

Fig. 1 First and second model of standing and walking by children with shortening of Achilles and m. triceps surae in children with MBD and ADHD

Material. The research material included 233 children aged from 2 to 15 . The research was conducted in the years 2010 - 2014. The examination of children was clinical, dynamic, podoscopic and radiological. In the course of the examination each orthopedic surgeon or physiotherapist should use a test and for the diagnosis of feet deformities the most suitable is the quick stretch test (QST).

Pathomorphology of flat and valgus deformity of feet. Tests. Mostly the pathology of the feet is connected with two characteristic changes 1/ laxity (Fig. 2) 


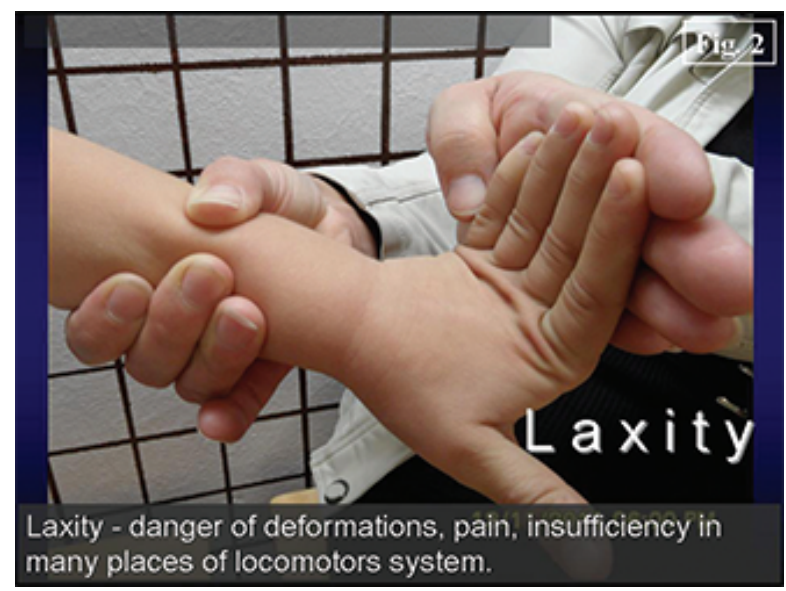

Fig. 2 Laxity - danger of deformations, pain, insufficiency in many places of locomotors system.

of soft tissues (laxity of collagen) and shortening (spastic or sub-spastic) of muscles $-\mathrm{m}$. gastrocnemius, $\mathrm{m}$. soleus ( $\mathrm{m}$. triceps surae), what is possible to check by quick stretch test (Fig. 3).

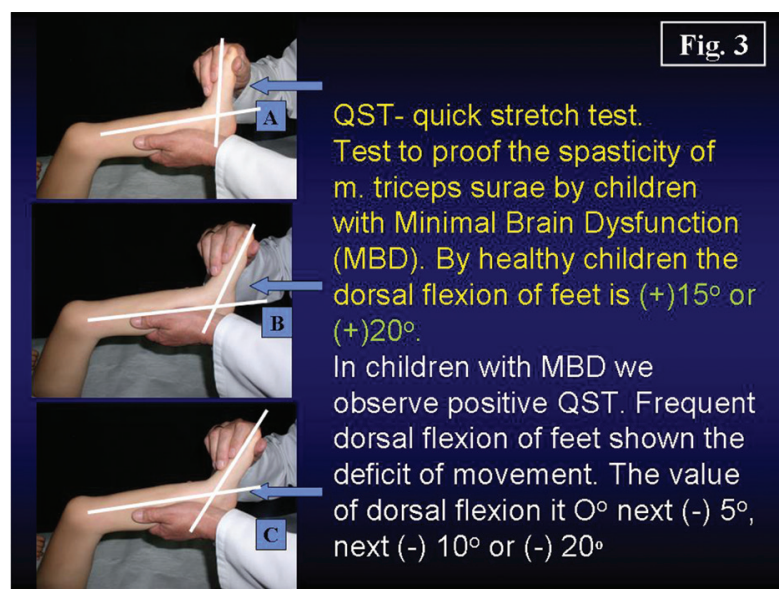

Fig. 3 QST- quick stretch test. Technique of test.

In gait dorsal flexion of feet is observed in every person and in every step. Due to the shortening of muscles and tendons this is impossible and the child walks with an equines position of the feet. If the coexistent laxity of joints is present - the dorsal flexion is possible only in the pronation position of feet. With time this "pronation position of feet" becomes stabilized and the foot presents valgus or plano - valgus deformity (Fig. 4a, 4b).
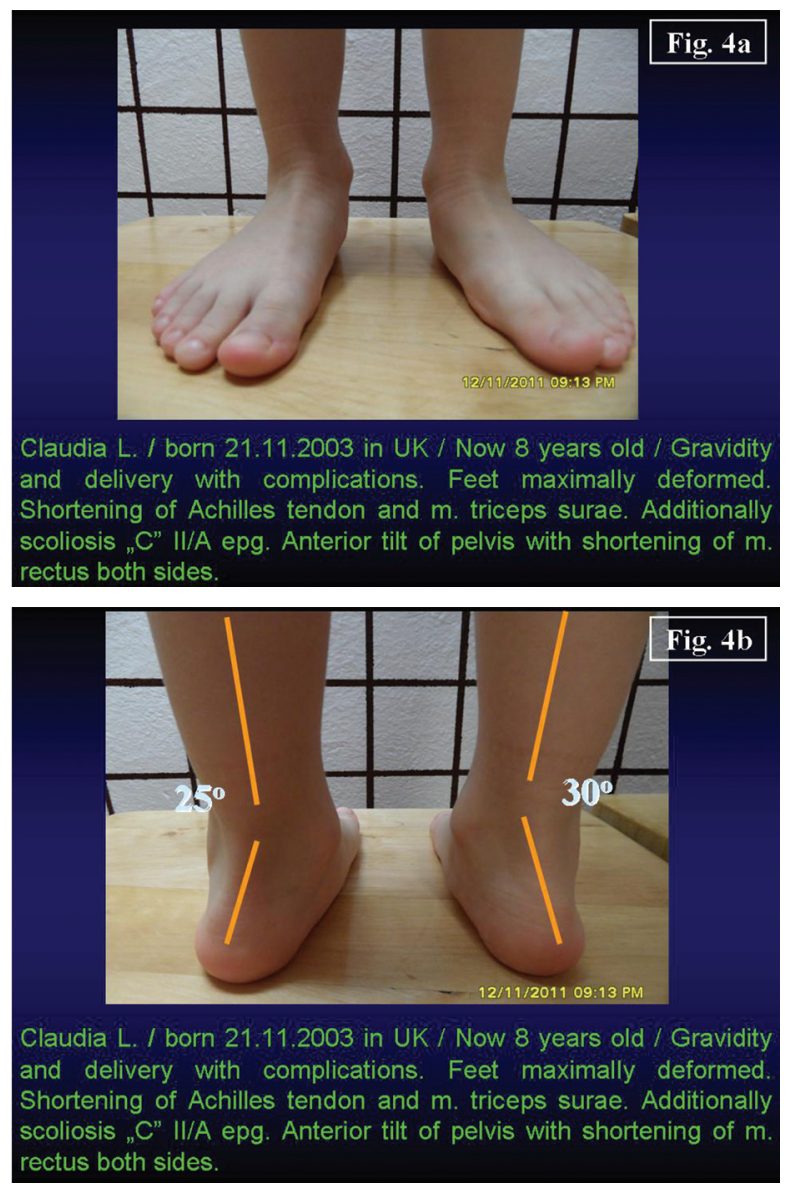

Fig. 4a, 4b Claudia L. / born 21.11.2003 in UK / Now 8 years old / Gravidity and delivery with complications. Feet maximally deformed. Shortening of Achilles tendon and m. triceps surae. Additionally scoliosis „C" II/A epg. Anterior tilt of pelvis with shortening of flexors of hips but a specially of $\mathrm{m}$. rectus both sides.

Other symptoms in ADHD and MBD children. In spite of feet deformities these children present different habits of behavior. These changes are the following: the children are often nervous, they are inclined to cry and their mood changes easily, they like being over active and jump. Consequently, permanent jumping may cause Perthes disease (Fig. 5).

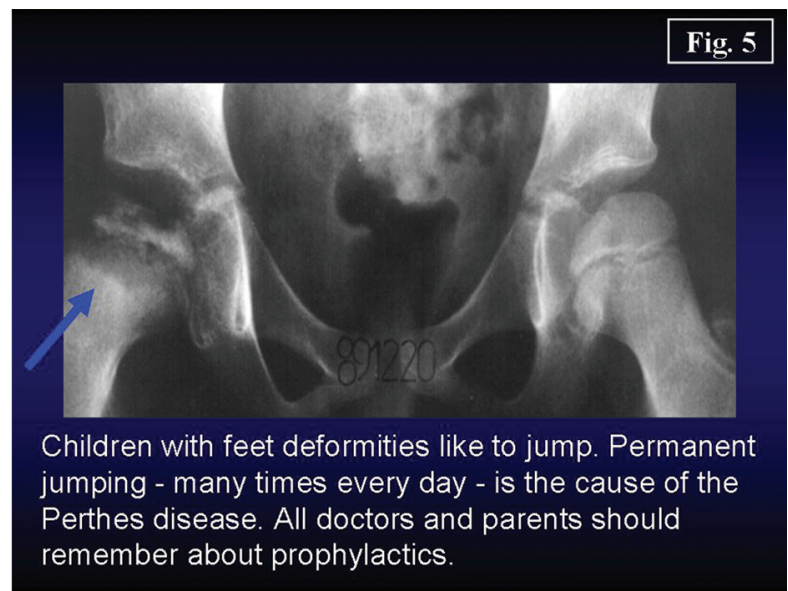

Fig. 5 Children with feet deformities like to jump. Permanent jumping - many times every day - is the cause of the Perthes disease. All doctors and parents should remember about prophylactics. 
Gynecologic, pediatric and neurologic observations. The question at issue is why there are so many cases of MBD and CP (in Lublin material this last group is $6 \%$ ).

The answer is the following: almost each gravidity and delivery present complications of some kind.

The following problems may occur during gravidity: bleeding, stress, high blood pressure, very low blood pressure, anemia, asthma and many others like multiple-gravidity and Twin Twin Transfusion Syndrome (TTTS).

The problems occurring during delivery may be as follows: premature delivery, prolonged delivery, Bracht maneuver delivery, vacuum extractor, umbilical cord strangulation, the cord tangled around the leg or arm or trunk of the child.

In all these cases asphyxia of brain followed by neurological symptoms may take place resulting in orthopedic changes in feet.

Accompanying deformity of knee, pelvis and trunk in children with foot deformities. Examples. In the photos we present examples of knee, pelvis and spine deformities in the children with feet deformities. The causes of these accompanied deformities is the same - sub spasticity of flexors group of muscles of knees and hips and laxity of joints (Fig. 6, 7a, 7b, 8, 9).

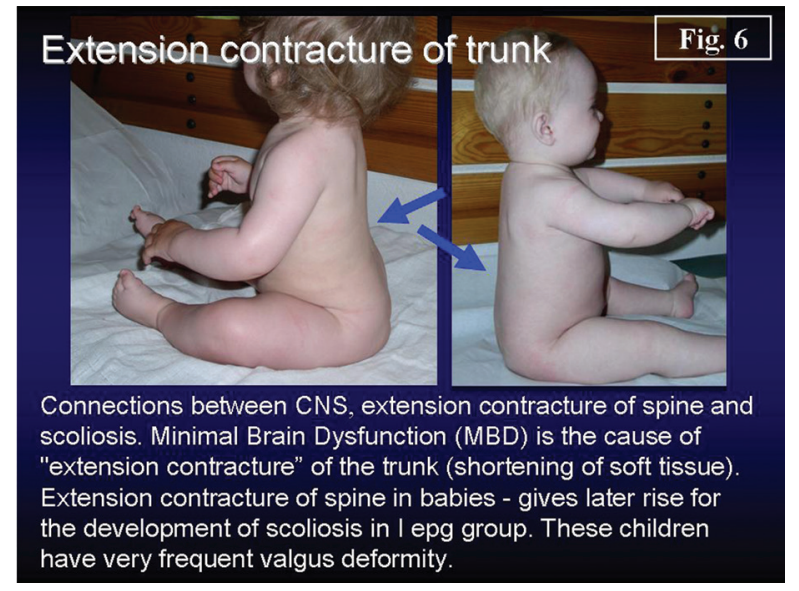

Fig. 6 Minimal Brain Dysfunction (MBD) is the cause of "extension contracture" of the trunk (shortening of soft tissue). Extension contracture of spine in babies - gives later rise for the development of scoliosis in I epg group. These children have very frequent valgus deformity.
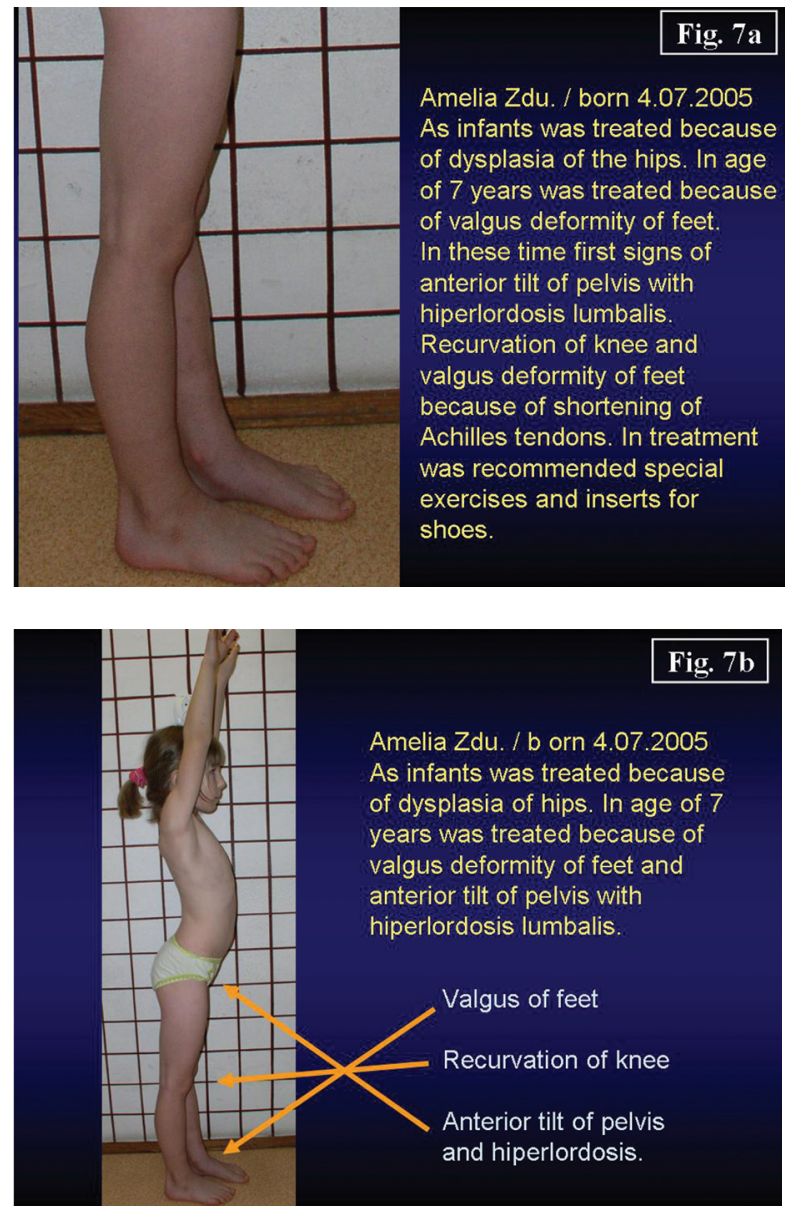

Fig. 7a, 7b Amelia Zdu. / born 4.07.2005. As infants was treated because of dysplasia of hips. In age of 7 years was treated because of valgus deformity of feet and anterior tilt of pelvis with hiperlordosis lumbalis.

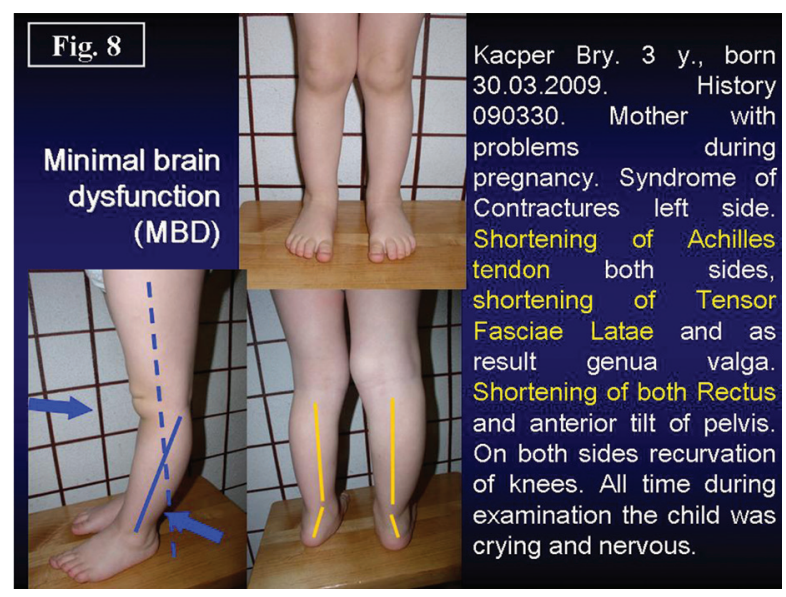

Fig. 8 Kacper Bry. 3 y., born 30.03.2009. Mother with problems during pregnancy. "Syndrome of Contractures" left side. Shortening of Achilles tendon both sides, shortening of Tensor Fasciae Latae and as result genua valga. Shortening of both Rectus and anterior tilt of pelvis. On both sides recurvation of knees. All time during examination the child was crying and nervous. 


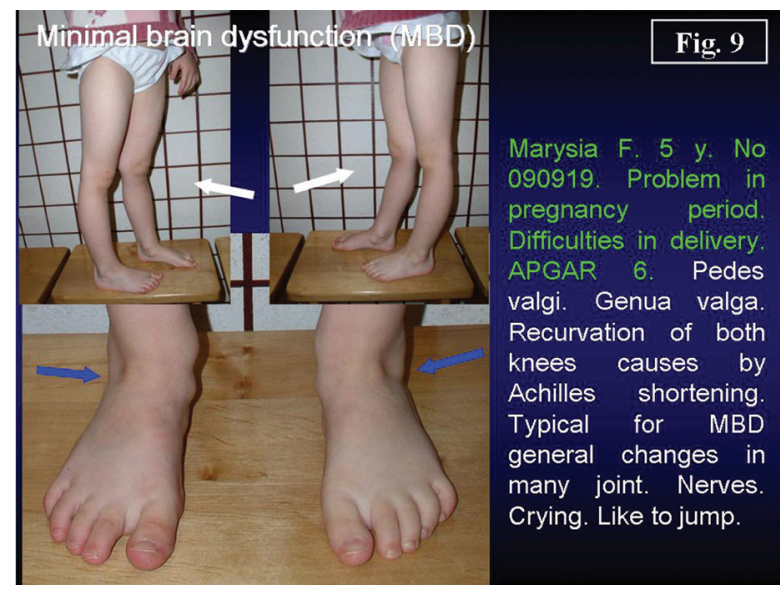

Fig. 9 Marysia F. 5 y. Born 19.09.2009. Problem in pregnancy period. Difficulties in delivery. APGAR 6. Pedes valgi, genua valga. Recurvation of both knees causes by Achilles shortening. Typical for MBD general changes in many joint. Nerves. Crying. Like to jump.

Physiotherapy in feet problems. Generally in physiotherapy only the stretching exercises are proper. Such therapy restore full movement of joints, restore symmetry in all movements of joints and next enable the proper growing of the child and normal function of all movements organs. In the photos you can see examples of feet, pelvis deformities and methods of treatment (Fig. 10, 11, 12, 13).

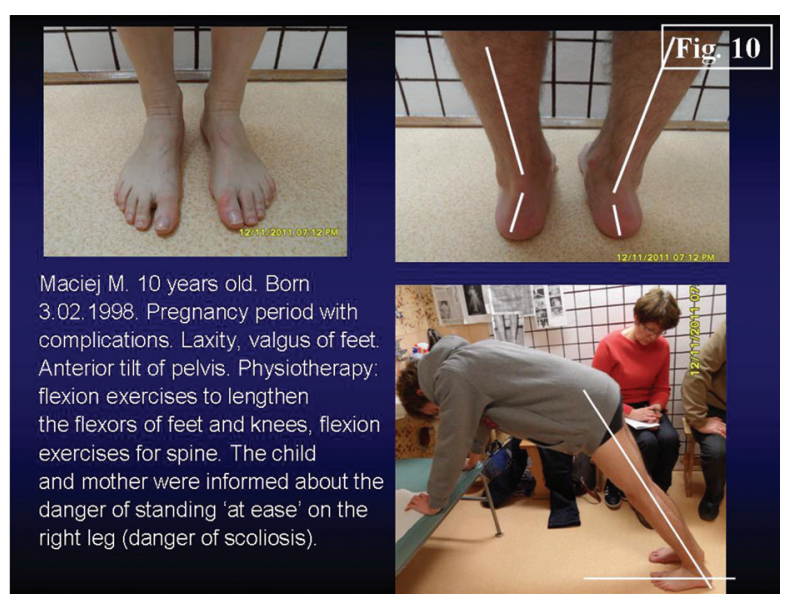

Fig. 10 Maciej M. 10 years old. Born 3.02.1998. Pregnancy period with complications. Laxity, valgus of feet. Anterior tilt of pelvis. Treatment: flexion exercises to lengthen the flexors of feet and knees. Flexion exercises for spine. The child and mother were informed about the danger of standing 'at ease' on the right leg (danger of scoliosis).

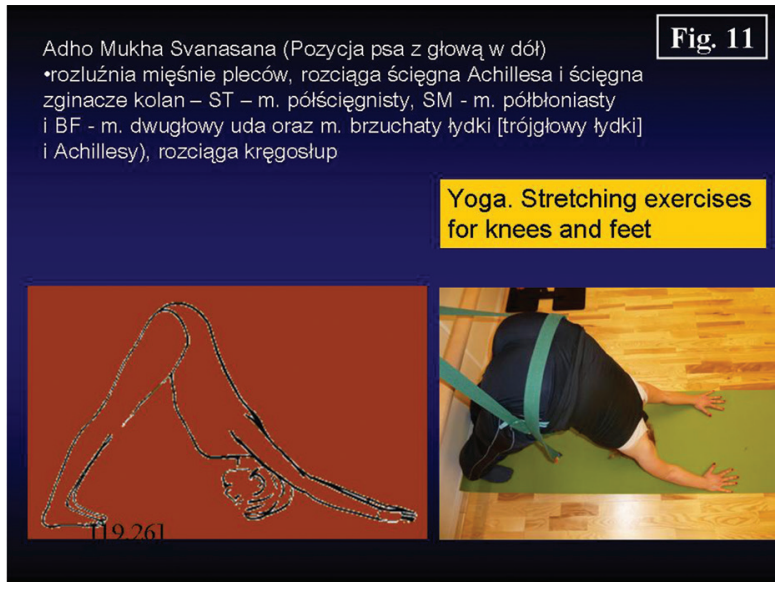

Fig. 11 Yoga. Stretching exercises for knees and feet.

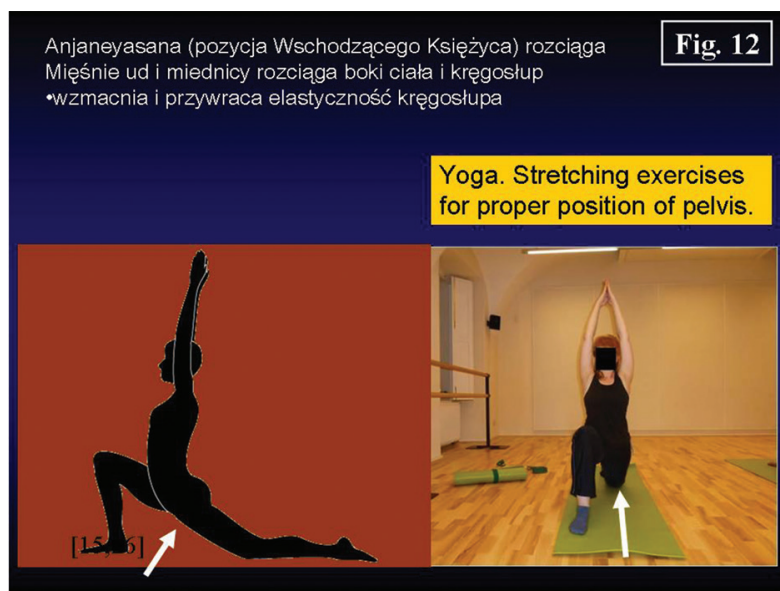

Fig. 12 Yoga. Stretching exercises for proper position of pelvis.

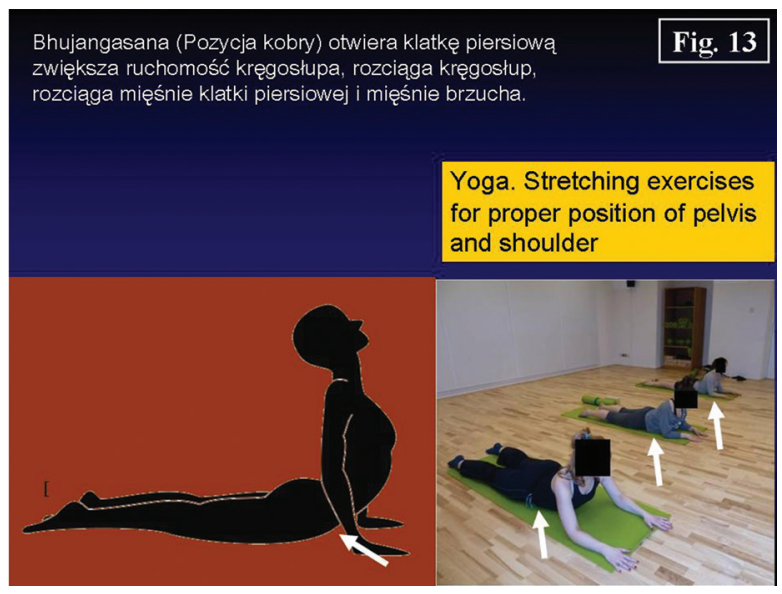

Fig. 13 Yoga. Stretching exercises for proper position of pelvis and shoulder

\section{Conclusions}

1/ Plat or plat-valgus deformity of the feet in children and youth are very frequent. Among the present generation of Polish children there is $6 \%$, but in general orthopedic practices we observe even 30 $\%$ of patients with feet deformities. 
2/ The causes of these deformities are mostly: minimal brain dysfunctions (MBD), attention deficit hyperactivity disorder (ADHD) or even full cerebral palsy $(\mathrm{CP})$ with spasticity additionally connected with general laxity of joints, that is laxity of collagen - in fascias and in capsules of joints.

3/ The plat or plat-valgus deformity develops mostly because of the shortening of Achilles tendon and shortening of $\mathrm{m}$. triceps surae which later - as causal factors - is connected is with function - walking.

4/ Proper therapy is only by stretching exercises, which should be introduced very early - even in children aged of $2-3$, before the fixation of feet deformities.

5/ For treatment we additionally use special inserts for shoes.
6/ The children with minimal brain dysfunctions (MBD) and attention deficit hyperactivity disorder (ADHD) are specially active and as prevention of Perthes disease we should stop the children from jumping.

\section{References:}

www.ortopedia.karski.lublin.pl www.karski.net.pl

\section{Correspondence address:}

Tomasz Karski, Vincent Pol University in Lublin Choiny 2

tmkarski@gmail.com 